DÉTERMINATION DE LA FONCTION DE STRUCTURE D'UNE CLASSE DE TARIF

\title{
L. D'HoOGE
}

Leuven (K.U.L.)

\section{Introduction DU Problème}

a) Le dépouillement statistique d'une classe de tarif hétérogène permet d'observer les fréquences relatives $q_{i}$ des contrats qui pendant une période de référence ont été frappés de $(i-\mathbf{I})$ sinistres.

On a:

$$
0 \leqslant q_{i} \leqslant \mathrm{I} \quad i=\mathbf{I}, 2, \ldots, m
$$

et

$$
\sum_{i=1}^{m} q_{i}=\mathrm{I}
$$

Ces fréquences déterminent dans l'espace $R^{m}$ un vecteur à $m$ composantes, que nous notons:

$$
\bar{Q}\left(q_{1}, q_{2}, \ldots, q_{m}\right)
$$

b) Une base de décomposition est constituée par un éventail fini de $n$ classes homogènes. Chaque classe homogène $C_{k}$ est définie par une variable aléatoire, dont la loi de probabilité $\bar{P}\left(\lambda_{k}\right)$ dépend du paramètre $\lambda_{k}$. Théoriquement ce paramètre peut être tout à fait général (un triplet réel p.e.); pour les applications pratiques traitées au paragraphe 4 , il suffit pourtant de considérer $\lambda_{k}$ comme un nombre réel.

Si $p_{i}\left(\lambda_{k}\right)$ représente la probabilité à priori d'avoir exactement $(i-\mathrm{I})$ sinistres dans la classe $C_{k}$, nous pouvons noter $\bar{P}\left(\lambda_{k}\right)$ par:

$$
\bar{P}\left(p_{1}\left(\lambda_{k}\right), p_{2}\left(\lambda_{k}\right), \ldots, p_{m}\left(\lambda_{k}\right)\right)
$$

On a:

$$
0 \leqslant p_{i}\left(\lambda_{k}\right) \leqslant \mathrm{I} \quad i=\mathrm{I}, 2, \ldots, m
$$

et

$$
\sum_{i=1}^{m} p_{i}\left(\lambda_{k}\right)=\mathrm{I}
$$


Nous supposons que les vecteurs $\bar{P}\left(\lambda_{k}\right)$ sont linéairement indépendants.

c) Résoudre l'hétérogénéité d'une classe de tarif consiste tout d'abord à choisir une base de décomposition, à représentation analytique connue, et puis à décomposer la classe de tarif par l'éventail des classes homogènes $C_{k}$ de cette base.

Mathématiquement le problème à résoudre consiste à vérifier s'il existe des nombres:

$$
\alpha_{k} \geqslant 0 \quad k=\mathrm{I}, 2, \ldots, n
$$

tel que l'on ait:

$$
\sum_{k=1}^{n} \alpha_{k}=\mathrm{I}
$$

et

$$
\bar{Q}=\sum_{k=1}^{n} \alpha_{k} \bar{P}\left(\lambda_{k}\right)
$$

$\alpha_{k}$ représente en fait la probabilité à priori qu'un risque quelconque appartient à la classe homogène $C_{k}$. Les nombres $\alpha_{k}$ définissent une variable aléatoire $\Lambda$ (variable hétérogénéité), caractérisant l'hétérogénéité de la classe de tarif considérée.

Nous avons par conséquent:

$$
\operatorname{Prob}\left(\Lambda=\lambda_{k}\right)=\alpha_{k} \quad k=\mathrm{I}, 2, \ldots, n
$$

Les $n$ couples $\left(\lambda_{k}, \alpha_{k}\right)$ peuvent être considérés comme une approximation de la fonction de structure:

$$
H(\lambda)=\operatorname{Prob}(\Lambda<\lambda)
$$

de la classe de tarif. Cette fonction est représentée graphiquement ci-dessous.

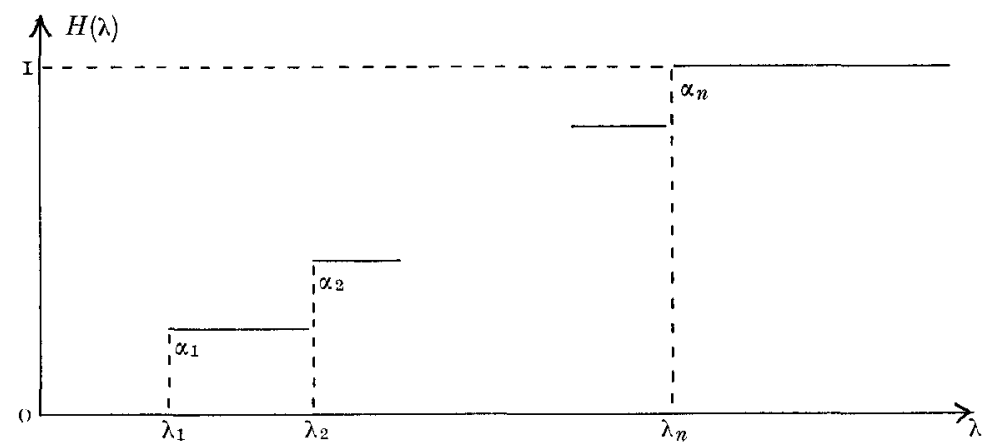

Fig. I 
d) Vérifier si une base de décomposition donnée convient ou ne convient pas, consiste à résoudre effectivement le système linéaire (I.5) de $m$ équations en $n$ inconnus $\alpha_{k}$ et à constater que la solution obtenue:

I) ou bien est telle que les contraintes (I.3) et (I.4) sont satisfaites. Le problème possède une solution et la base de décomposition choisie est adéquate;

2) ou bien la solution est telle que ces contraintes ne sont pas vérifiées. Le problème n'a pas de solution et la base de décomposition choisie n'est pas adéquate. Dans ce cas on pourrait modifier la base initialement choisie et recommencer la vérification.

L'expérience montre pourtant, qu'il est pratiquement impossible de construire ,au hasard" une base de décomposition adéquate ou de détecter la non-existence d'une telle base, si celle-ci comporte plus de 3 classes homogènes.

L'objet de la communication au roème Colloque d'A.S.T.I.N. est de montrer qu'un algorithme simple permet de construire progressivement, à partir d'une base de décomposition initiale et non adéquate, des bases de plus en plus adéquates en manière telle d'obtenir finalement une base adéquate (si une telle base existe).

L'approche géométrique du problème, développé au paragraphe 2, facilitera la recherche d'un tel algorithme.

\section{Remarque}

Contrairement à l'hypothèse admise, que l'on a un nombre fini de classes homogènes, certains actuaires Bichsel [I], Coppini [2], Delaporte [3], et d'autres ont envisagé de représenter la fonction de structure $H(\lambda)$ par une fonction continue Gamma de façon à pouvoir profiter des facilités de calcul.

Cette hypothèse implique l'admission d'un nombre infini de sousclasses homogènes, alors que l'effectif même de la classe de tarif est fini. D'autre part pour passer au calcul effectif on est obligé de remplacer la fonction de structure continue par une fonction en escalier.

Cette note montrera que l'hypothèse de la continuité de la fonction de structure n'est pas une condition nécessaire. 


\section{Interprétation GÉometrigue}

a) Les $n$ vecteurs $\bar{P}\left(\lambda_{k}\right)$ de la base de décomposition engendrent dans l'espace $R^{m}$ un cône polyhédrique convexe.

Désignons par $\bar{u}(\mathrm{I}, \mathrm{I}, \ldots, \mathrm{I})$ le vecteur unitaire de l'espace $R^{m}$ et $\operatorname{par} \bar{e}_{i}(0,0, \ldots, \mathrm{I}, \ldots, 0), i$ variant de I à $m$, les $m$ vecteurs unitaires du $m$-èdre de référence.

On a d'après (I.2):

$\forall k:$

$$
\bar{u} \bar{P}\left(\lambda_{k}\right)=\sum_{i=1}^{m} p_{i}\left(\lambda_{k}\right)=\mathrm{I}
$$

Par conséquent les $n$ vecteurs $\bar{P}\left(\lambda_{k}\right)$ induisent dans le simplexe, formé par l'intersection de l'hyperplan $\bar{e}_{1} \bar{e}_{2} \ldots \bar{e}_{m}$ et l'orthant positif de l'espace $R^{m}$, un ensemble convexe $\mathfrak{S}$ sous-tendu par les vecteurs:

$$
\bar{P}\left(\lambda_{1}\right), \bar{P}\left(\lambda_{2}\right), \ldots, \bar{P}\left(\lambda_{k}\right), \ldots, \bar{P}\left(\lambda_{n}\right)
$$

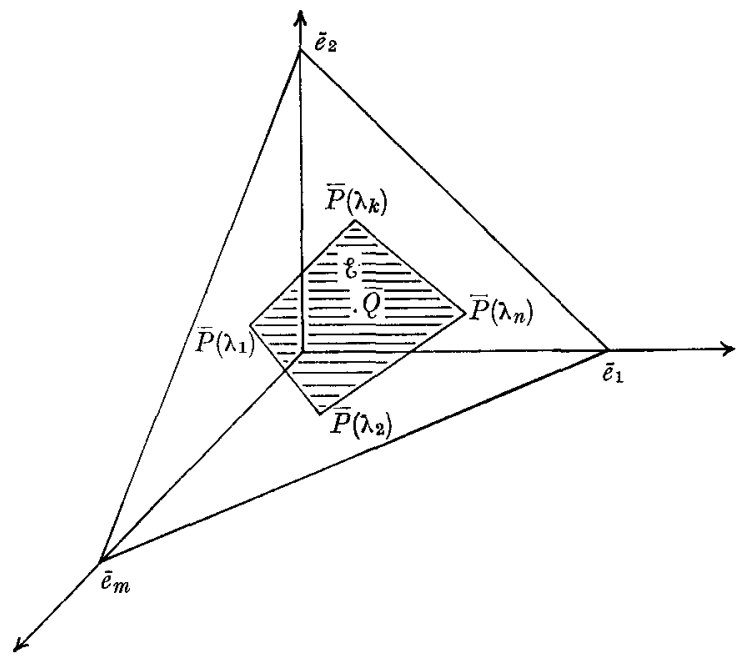

Fig. 2

b) Le vecteur $\bar{Q}$ représentatif de la classe de tarif donnée appartient au même simplexe, car on a d'après (I.I):

$$
\bar{u} \bar{Q}=\sum_{i=1}^{m} q_{i}=\mathrm{I}
$$


c) La base de décomposition donnée est adéquate si et seulement si le vecteur $\bar{Q}$ est situé à l'intérieur ou sur le bord de l'ensemble convexe $\mathfrak{H}$.

Notre objectif est de prouver que nous pouvons résoudre pratiquement le problème de la décomposition d'une classe de tarif, si nous disposons d'un algorithme permettant de construire autour du vecteur $\bar{Q}$ donnée un ensemble convexe $\mathfrak{h}$, sous-tendu par des vecteurs $\bar{P}\left(\lambda_{k}\right)$.

\section{Algorithme de RÉsolution}

(Nous supposons connu la théorie élémentaire de la programmation linéaire. On pourra se référer aux livres classiques de Gale [8] et de Simonnard [9].

L'algorithme que nous proposons comporte deux stades:

Stade $I$ : vérifier si une base de décomposition donnée est adéquate.

Cette vérification comporte la résolution d'un programme linéaire. Si la base est adéquate le problème est résolu; sinon on passe au stade 2.

Stade 2: améliorer la base de décomposition non adéquate, obtenue au stade précédent, par l'introduction d'une classe homogène supplémentaire.

Cette amélioration, si elle est possible, s'obtient par post-optimation du programma linéaire résolu au stade I. Après cette opération on retourne au stade $\mathrm{I}$.

3.I. Stade I: Vérification d'une base de décomposition.

a) Franckx [7] a montré que le problème suivant:

Chercher des nombres $\alpha_{k} \geqslant 0$, tel que l'on ait:

$$
\bar{Q}=\sum_{k=1}^{n} \alpha_{k} \bar{P}\left(\lambda_{k}\right)
$$

peut se transformer en un programme linéaire.

La démonstration s'obtient assez facilement. Posons à priori:

$$
\bar{Y}=\left(y_{1}, y_{2}, \ldots, y_{m}\right)
$$


et considérons le programme linéaire (I) suivant:

$$
\text { (I) }\left\{\begin{array}{l}
\bar{Q}=\sum_{k=1}^{n} \alpha_{k} \overrightarrow{P\left(\lambda_{k}\right)}+\bar{Y} \\
\alpha_{1} \geqslant 0, \alpha_{2} \geqslant 0, \ldots, \alpha_{n} \geqslant 0 \\
y_{1} \geqslant 0, \quad y_{2} \geqslant 0, \ldots, \quad y_{m} \geqslant 0 \\
\text { maximiser: } \quad z=\sum_{k=1}^{n} \alpha_{k}
\end{array}\right.
$$

Le système linéaire (3.I) aura une solution non négative si et uniquement si toutes les composantes de $\bar{Y}$ sont nulles. Dans ce cas on a également:

$$
\sum_{k=1}^{m} y_{k}=0
$$

et les valeurs des $\alpha_{k}$ donnent la solution non négative de (3.1). Mais en multipliant la contrainte (3.2) par le vecteur unitaire $\bar{u}$, on trouve:

$$
\bar{u} \bar{Q}=\sum_{k=1}^{n} \alpha_{k} \bar{u} \bar{P}\left(\lambda_{k}\right)+\bar{u} \bar{Y}
$$

Or, d'après (2.2) on a:

$$
\bar{u} \bar{Q}=\mathrm{I}
$$

et d'après (2.I):

$\forall k$

$$
\bar{u} \bar{P}\left(\lambda_{k}\right)=\mathbf{I}
$$

(3.5) devient finalement:

$$
\sum_{k=1}^{n} \alpha_{k}=\mathrm{I}-\sum_{k, 1}^{m} y_{k}
$$

Par conséquent le minimum de $\sum^{m} y_{k}$ correspond d'après (3.6) au maximum de:

$$
z=\sum_{k=1}^{n} \alpha_{k}
$$

et on peut dès lors introduire le problème de la vérification d'une base de décomposition donnée sous la forme opérationnelle $(I)$.

b) La solution de programme linéaire $(I)$ est donnée par la méthode du simplexe ou par une de ses variantes. 
Le tableau de départ sous forme canonique est le suivant:

\begin{tabular}{|c|c|c|c|c|c|c|c|}
\hline & $\bar{e}_{1}$ & $\bar{e}_{2}$ & $\ldots \ldots \bar{e}_{m}$ & $\left.\overline{P(} \lambda_{1}\right)$ & $\bar{P}\left(\lambda_{2}\right)$ & $\ldots \bar{P}\left(\lambda_{n}\right)$ & \\
\hline $\bar{e}_{1}$ & I & $o$ & $\ldots \ldots o$ & $p_{1}\left(\lambda_{1}\right)$ & $p_{1}\left(\lambda_{2}\right)$ & $\cdots p_{1}\left(\lambda_{n}\right)$ & $q_{1}$ \\
\hline $\bar{e}_{2}$ & o & I & $\ldots \ldots o$ & $p_{2}\left(\lambda_{1}\right)$ & $p_{2}\left(\lambda_{2}\right)$ & $\cdots p_{2}\left(\lambda_{n}\right)$ & $q_{2}$ \\
\hline & . & $B_{0}$ & $\ldots$ & $\cdot \cdot$ & . . . & . . . . . . & . . \\
\hline $\bar{e}_{m}$ & o & o & $\ldots I$ & $p_{m}\left(\lambda_{1}\right)$ & $p_{m}\left(\lambda_{2}\right)$ & $\ldots p_{m}\left(\lambda_{n}\right)$ & $q_{m}$ \\
\hline$c_{j}$ & o & 0 & . . . o & I & $\mathbf{I}$ & I & $z=0$ \\
\hline
\end{tabular}

La ligne marginale contient les coefficients $c_{j}$ avec lesquels les variables $\alpha$ et $y$ apparaissent dans la fonction objective $z$. Les vecteurs $\bar{e}_{1}, \bar{e}_{2}, \ldots, \bar{e}_{m}$ constituent la base initiale $B_{0}$ du programme, qui admet la solution initiale suivante:

$$
\begin{aligned}
& y_{1}=q_{1}, \quad y_{2}=q_{2}, \ldots, y_{m}=q_{m} \\
& \alpha_{1}=0, \quad \alpha_{2}=0, \ldots, \alpha_{n}=0
\end{aligned}
$$

Pour cette solution la fonction objective $z$ est égale à 0 .

Géometriquement cela signifie que l'ensemble convexe $\mathfrak{h}$ construit initialement autour du vecteur $\bar{Q}$ ne contient que les vecteurs $\bar{e}_{k}$. La méthode du simplexe consiste précisément à construire au cours d'une itération un nouvel ensemble convexe $\mathfrak{S}^{\prime}$ autour de $\bar{Q}$ ne différant de $\mathfrak{h}$ que par un seul vecteur et de manière telle que:

I) $\widetilde{Q}$ reste à l'intérieur de $\mathfrak{\mathfrak { Q }}^{\prime}$;

2) z a augmenté.

c) Si la solution du programme est telle que la fonction objective $z$ atteint effectivement sa valeur maximale I cela veut dire que l'on est parvenu à construire autour du vecteur $\bar{Q}$ un ensemble convexe ne contenant plus aucun vecteur $\bar{e}_{k}$ et constitué donc uniquement de vecteurs $\vec{P}\left(\lambda_{k}\right)$.

On a trouvé dès lors une base de décomposition adéquate, qui contient comme on peut s'en rendre compte au maximum $m$ lois homogènes. Le , dosage" des lois se lit dans la dernière colonne du tableau. 
3.2. Stade 2: Amélioration d'une base de décomposition non adéquate

a) Si la solution du programme linéaire $(I)$ est telle que $z$ n'atteint pas sa valeur maximale $I$, l'ensemble convexe $\mathfrak{h}$, construit autour de $\bar{Q}$, contient encore un certain nombre de vecteurs unitaires $\bar{e}_{k}$ du $m$-èdre de référence.

Nous supposons que $\mathfrak{H}$ contient actuellement d'une part $r$ vecteurs $\vec{P}\left(\lambda_{k}\right)$

$$
\bar{P}\left(\lambda_{j_{1}}\right), \bar{P}\left(\lambda_{j_{2}}\right), \ldots, \bar{P}\left(\lambda_{j_{r}}\right) \quad j_{k} \varepsilon \mathrm{I}, 2, \ldots, n
$$

et d'autre part, en complément les $(m-r)$ vecteurs $e_{k}$ :

$$
\bar{e}_{i_{1}}, \bar{e}_{i_{2}}, \ldots, \bar{e}_{i_{m-r}} \quad i_{k} \varepsilon \mathrm{I}, 2, \ldots, m
$$

A ce stade le tableau du simplexe se présente de la façon suivante:

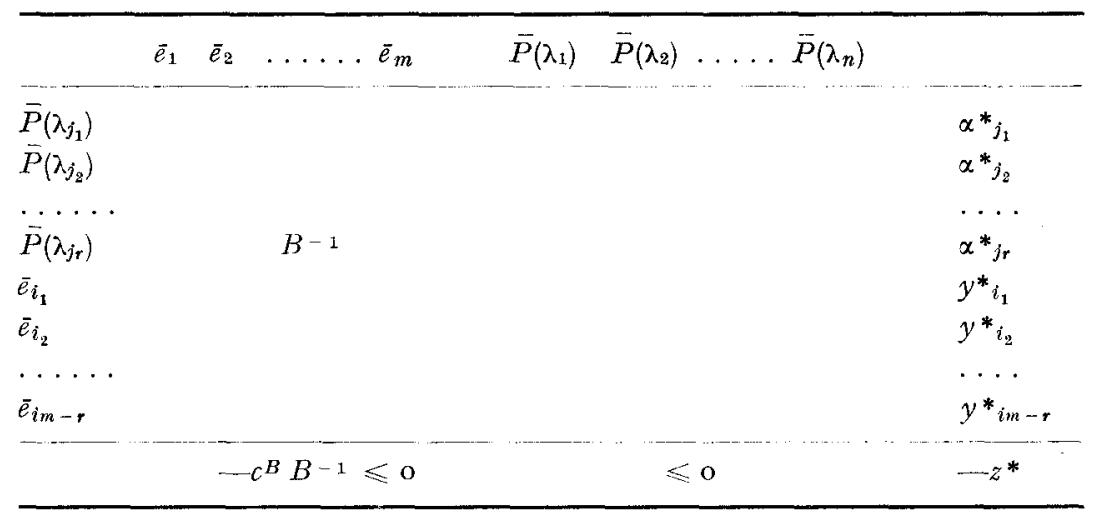

Ĺa base actuelle $B$ du programme est constituée par les vecteurs:

$$
\left.\left.\bar{P}\left(\lambda_{j_{1}}\right), \bar{P}\left(\lambda_{j_{2}}\right), \ldots, \bar{P}\right) \lambda_{j_{r}}\right)
$$

de la base de décomposition donnée et par les vecteurs:

du $m$-èdre de référence.

$$
\bar{e}_{i_{1}}, \bar{e}_{i_{2}}, \ldots, \bar{e}_{i_{n-r}}
$$

(L'ordre dans lequel ces vecteurs se présentent dans la base $B$ n'est pas nécessairement comme indiqué dans le tableau; cet ordre dépend de la séquence d'itérations effectuées pour passer de la base initiale $B_{0}$ à la base actuelle $B$ ). 
$c^{B}$ représente un vecteur ligne dont les $m$ composantes sont les coefficients avec lesquels les variables de base apparaissent dans l'expression (3.4) de la fonction objective $z$ (c.a.d. I pour une variable $\alpha$ et o pour une variable $y$ ).

Le tableau contient, comme indiqué, la matrice inverse $B^{-1}$ de la base actuelle $B$. Le vecteur $-\mathrm{c}^{B} B^{-1}$ est situé dans le tableau endessous de $B^{-1}$.

Ce programme admet la solution suivante:

$$
\begin{array}{ll}
y_{i_{1}}=y_{i_{1}}^{*}, & y_{i_{2}}=y_{i_{2}}^{*}, \quad \ldots, \quad y_{i_{m-r}}=y_{i_{m-r}}^{*} \\
\alpha_{j_{1}}=\alpha_{j_{1}}^{*}, & \alpha_{j_{2}}=\alpha_{j_{2}}^{*}, \ldots, \quad \ldots, \alpha_{j_{r}}=\alpha_{j_{r}}^{*}
\end{array}
$$

et on a:

$$
z=z^{*}<\mathrm{I}
$$

Comme tous les cofficients de la ligne marginale sont négatifs ou nuls, on ne peut plus augmenter la fonction objective $z$.

b) Dans ce cas il est impossible de construire une base adéquate, au moyen des $n$ vecteurs $\bar{P}\left(\lambda_{k}\right)$ de la base de décomposition donnée. Il faut introduire une nouvelle classe homogène $C_{n+1}$ définie par sa loi de probabilité $\widetilde{P}\left(\lambda_{n+1}\right)$.

Vérifier si la nouvelle base de décomposition formée par les $(n+\mathrm{I})$ vecteurs:

$$
\bar{P}\left(\lambda_{1}\right), \bar{P}\left(\lambda_{2}\right), \ldots, \bar{P}\left(\lambda_{n}\right), \bar{P}\left(\lambda_{n+1}\right)
$$

est adéquate, consiste à résoudre le nouveau programme linéaire (II) suivant:

$$
\text { (II) }\left\{\begin{array}{l}
\bar{Q}=\sum_{k=1}^{n+1} \alpha_{k} \bar{P}\left(\lambda_{k}\right)+\bar{Y} \\
\alpha_{1} \geqslant 0, \alpha_{2} \geqslant 0, \ldots, \alpha_{n} \geqslant 0, \alpha_{n+1} \geqslant 0 \\
y_{1} \geqslant 0, y_{2} \geqslant 0, \ldots, y_{m} \geqslant 0 \\
\text { maximiser: } \quad z=\sum_{k=1}^{n+1} \alpha_{k}
\end{array}\right.
$$

On constate que ce nouveau programme s'obtient par addition d'une variable $\alpha_{n+1}$ au programme primitif $(I)$. Cette addition s'accompagne de l'addition au tableau du simplexe d'une colonne $\bar{P}\left(\lambda_{n+1}\right)$ et d'un coefficient $c_{n+1}=\mathrm{I}$ dans la ligne marginale. 
Dans ce cas le programme maximal de base antérieur reste un programme de base du programme modifié, mais ce programme peut ne plus être maximal.

Pour prendre une décision en ce qui concerne l'introduction du vecteur $\bar{P}\left(\lambda_{n+1}\right)$ dans la base de décomposition, il faut calculer:

$$
\left.r_{n+1}=c_{n+1}-c^{B} B^{-1} \overline{P(} \lambda_{n+1}\right)=\mathrm{I}-c^{B} B^{-1} \bar{P}\left(\lambda_{n+1}\right)
$$

Cette grandeur se calcule facilement, puisqu'on connait le vecteur $c^{B} B^{-1}$ (méthode de la matrice inverse).

I) Si $r_{n+1}<0$, le programme primitif est aussi un programme maximal du programme modifié (II) et l'introduction de $\bar{P}\left(\lambda_{n+1}\right)$ n'augmentera pas la fonction objective $z$.

2) Si $r_{n+1}=$ o le vecteur $\bar{P}\left(\lambda_{n+1}\right)$ fait déjà partie de la base de décomposition.

3) Si $r_{n+1}>0$ on augmente la fonction objective $z$ en introduisant $\bar{P}\left(\lambda_{n+1}\right)$ dans la base de décomposition (et dans la base $B$ du programme)

Dans ce cas, comme on dispose de $B^{-1}$, l'algorithme de la matrice inverse peut être appliqué directement à partir du dernier tableau maximal du problème primitif $(I)$, complété par une $(n+\mathrm{I})$ ème colonne. Cette colonne contiendra le vecteur $B^{-1} \bar{P}\left(\lambda_{n+1}\right)$ et la grandeur $r_{n+1}$ dans la ligne marginale.

\subsection{Mise en route de l'algorithme}

L'algorithme peut toujours être mis en route. Il suffit d'introduire une seule classe homogène $C_{\mathbf{1}}$, définie par sa loi de probabilité $P\left(\lambda_{1}\right)$. On vérifie qu'on a toujours:

$$
r_{1}=\mathrm{I}>0
$$

car au départ, on a:

$$
c^{B}=(0,0, \ldots, 0)
$$

Il suffit donc, pour manier la méthode, de garder uniquement en mémoire la matrice $B^{-1}$ et le vecteur $c^{B} B^{-1}$, ainsi que la colonne „solution" évidemment. 


\section{Exemples Numérigues}

\section{Exemple I}

Delaporte [3] a observé pour une classe de tarif automobile la répartition des nombres probables de sinistres par véhicule en I958.

Les colonnes ( $\mathrm{I}$ ) et (2) du tableau ci-dessous donnent les résultats de l'enquête.

\begin{tabular}{|c|c|c|c|}
\hline $\begin{array}{c}\text { Nombres de sinistres } \\
\text { observés } \\
\text { (I) }\end{array}$ & $\begin{array}{l}\text { Nombre observé } \\
\text { de véhicules } \\
(2)\end{array}$ & $\begin{array}{l}\bar{Q} \\
(3)\end{array}$ & $\begin{array}{c}\sum_{k} \alpha_{k} \bar{P}\left(\lambda_{k}\right) \\
\quad(4)\end{array}$ \\
\hline 0 & 774 & 0,580210 & 0,580210 \\
\hline I & 375 & 0,28 I 109 & 0,281109 \\
\hline 2 & 120 & 0,089955 & 0,089955 \\
\hline 3 & 40 & 0,029985 & 0,029985 \\
\hline 4 & I 5 & 0,011244 & 0,011244 \\
\hline 5 & 5 & 0,003748 & $0,00374^{8}$ \\
\hline 6 & 2 & 0,001499 & 0,001275 \\
\hline 7 & I & $0,00075^{\circ}$ & $0,00075^{\circ}$ \\
\hline 8 & I & $0,00075^{\circ}$ & $0,00075^{\circ}$ \\
\hline 9 & I & $0,00075^{\circ}$ & $0,00075^{\circ}$ \\
\hline
\end{tabular}

La clécomposition de $\bar{Q}$ (colonne 3 ) par des lois binomiales $\bar{P}\left(\lambda_{k}\right)$ de composantes:

$$
p_{i}\left(\lambda_{k}\right)=C_{9}^{i-1} \lambda_{k}^{i-1}\left(\mathrm{I}-\lambda_{k}\right)^{9-i+1} \quad i=\mathrm{I}, 2, \ldots, \text { I0 }
$$

a donné les résultats suivants:

\begin{tabular}{lll}
\hline$k$ & $\lambda_{k}$ & $\alpha_{k}$ \\
\hline $\mathrm{I}$ & 0,01 & $0, \mathbf{I} 438$ \\
2 & 0,05 & 0,08428 \\
3 & 0,06 & 0,6784 \\
4 & 0,24 & 0,02630 \\
5 & 0,25 & 0,06382 \\
6 & 0,70 & 0,001450 \\
7 & 0,71 & 0,0004828 \\
8 & 0,93 & 0,0009319 \\
9 & 0,94 & 0,0003206 \\
\hline
\end{tabular}

On peut vérifier que: $z=0,9998$.

La colonne (4) donne les composantes du vecteur $\Sigma \alpha_{k} \bar{P}\left(\lambda_{k}\right)$. 


\section{Exemple 2}

Coppini [2] donne la distribution de 8.695 femmes d'après le nombre de cas de maladie en Ig6o.

\begin{tabular}{crcc}
\hline Nombre de cas & Données observées & $\bar{Q}$ & $\sum_{k} \alpha_{k} \bar{P}\left(\lambda_{k}\right)$ \\
\hline 0 & $574 \mathrm{I}$ & 0,660265 & 0,660265 \\
$\mathrm{I}$ & $\mathrm{I} 890$ & $0,2 \mathrm{I} 7366$ & $0,2 \mathrm{I} 7366$ \\
2 & 662 & $0,076 \mathrm{I} 36$ & $0,076 \mathrm{I} 36$ \\
3 & 253 & 0,029097 & 0,029097 \\
4 & 94 & $0,0108 \mathrm{II}$ & $0,0108 \mathrm{II}$ \\
5 & 38 & 0,004370 & $0,004 \mathrm{I} 36$ \\
6 & $\mathrm{I} 2$ & 0,001380 & 0,001380 \\
7 & 4 & 0,000460 & 0,000374 \\
8 & $\mathrm{I}$ & $0,0001 \mathrm{I} 5$ & $0,000 \mathrm{II}$ \\
\hline
\end{tabular}

La décomposition de $\bar{Q}$ par des lois binomiales $\bar{P}\left(\lambda_{k}\right)$ de composantes:

$$
p_{i}\left(\lambda_{k}\right)=C_{8}^{i-1} \lambda_{k}^{i-1}\left(\mathrm{I}-\lambda_{k}\right)^{8-i+1} \quad i=\mathrm{r}, 2, \ldots, 9
$$

a donné les résultats suivants

\begin{tabular}{lll}
\hline$k$ & $\lambda_{k}$ & $\alpha_{k}$ \\
\hline $\mathrm{I}$ & 0,02 & $0,356 \mathrm{I}$ \\
2 & 0,03 & 0,3749 \\
3 & $0, \mathrm{I} 5$ & 0,08732 \\
4 & $0, \mathrm{I} 6$ & $0, \mathrm{I} 592$ \\
5 & 0,43 & $0,02 \mathrm{I} 9 \mathrm{r}$ \\
6 & 0,87 & 0,00002795 \\
7 & 0,88 & 0,0002228 \\
\hline
\end{tabular}

On a: $\quad z=0,9997$

\section{Exemple 3}

La méthode exposée reste d'application dans le cas où la variable observée est la hauteur du sinistre, qui donne le montant aléatoire qui sera payé, lorsqu'un sinistre est survenu.

La composante $q_{i}$ du vecteur $\bar{Q}$ représente alors la fréquence relative des montants compris entre $m_{i}$ et $m_{i+1}$. La composante $p_{i}\left(\lambda_{k}\right)$ du vecteur $\bar{P}\left(\lambda_{k}\right)$ représente la probabilité à priori qu'un montant soit compris entre $m_{i}$ et $m_{i+1}$, si le risque appartient à la classe homogène $C_{k}$. 
Dropkin [6] donne la répartition de l'indemnité payée en I96I aux 24613 assurés, touchés d'une incapacité de travail permanente. Après regroupement des données et un changement d'échelle, nous retenons la distribution suivante.

\begin{tabular}{|c|c|c|}
\hline $\begin{array}{l}\text { Classes de fréquence } \\
\text { de } m_{i} \grave{a} m_{i+1} \text { (non compris) }\end{array}$ & Nombre observé & $\bar{Q}$ \\
\hline $0-2$ & 434 & 0,02 I I I 5 I I \\
\hline $2-3.5$ & I 106 & 0,05380948 \\
\hline $3,5-5$ & I958 & $0,09526 \mathrm{I} 26$ \\
\hline $5-12,5$ & 6951 & 0,3381823 \\
\hline $12,5-20$ & 4203 & $0,2044^{857}$ \\
\hline $20-27,5$ & 2635 & O, I 281989 \\
\hline $27,5-35$ & 1596 & 0,07764912 \\
\hline $35-42,5$ & 867 & 0,042 I I 8 I 57 \\
\hline $4^{2,5}-5^{\circ}$ & 422 & 0,02053128 \\
\hline $50-57,5$ & 225 & o,orog4677 \\
\hline $57,5-65$ & 88 & $0,00428 I_{4} 0_{5}$ \\
\hline $65-72,5$ & 35 & 0,001702832 \\
\hline $72,5-82,5$ & I9 & 0,0009243943 \\
\hline $82,5-95$ & 9 & 0,0004378710 \\
\hline $95-\mathbf{I} 7,5$ & 6 & $0,000291914^{\circ}$ \\
\hline
\end{tabular}

Nous décomposons $\bar{Q}$ par des lois Gamma $\bar{P}\left(\lambda_{k}\right)$. Notons par $F\left(m, \lambda_{k}\right)$ la fonction de répartion de la hauteur du sinistre dans la classe $C_{k}$. Nous avons:

$F\left(m, \lambda_{k}\right)=$ probabilité que la hauteur du sinistre soit inférieur à $m$

$$
=\mathrm{I}-e^{-m}\left(\mathrm{I}+\frac{m}{\mathrm{I} !}+\frac{m^{2}}{2 !}+\ldots+\frac{m^{\lambda^{k}-1}}{\left.\left(\lambda_{k}-\mathrm{I}\right) !\right)}\right)\left(\lambda_{k} \text { entier }\right)
$$

et par conséquent:

$$
p_{i}\left(\lambda_{k}\right)=F\left(m_{i+1}, \lambda_{k}\right)-F\left(m_{i}, \lambda_{k}\right)
$$

Cette décomposition a donné le résultat suivant:

\begin{tabular}{cll}
\hline$k$ & $\lambda_{k}$ & \multicolumn{1}{c}{$\alpha_{k}$} \\
\hline 1 & 2 & 0,00002350406 \\
2 & 3 & 0,05489099 \\
3 & 6 & 0,1109684 \\
4 & 7 & 0,3342800 \\
5 & 18 & 0,1454055 \\
6 & I9 & 0,1771584 \\
7 & 32 & 0,1004197
\end{tabular}




\begin{tabular}{rrl}
\hline$k$ & $\lambda_{k}$ & \multicolumn{1}{c}{$\alpha_{k}$} \\
\hline 8 & 33 & 0,03585424 \\
9 & 47 & 0,006646244 \\
I0 & 48 & 0,02876527 \\
II & 63 & 0,002787897 \\
I2 & 64 & 0,001567089 \\
I3 & 81 & 0,0009361454 \\
I4 & 82 & 0,00002404650 \\
I5 & I05 & 0,000272505 I \\
\hline
\end{tabular}

Comme $z=\mathrm{I}$, on a nécessairement:

$$
\left.\bar{Q}=\sum_{k} \alpha_{k} \overline{P(} \lambda_{k}\right)
$$

Nous remercions vivement W. van Gampelaere de l'aide qu'il nous a apporté pour la programmation et les calculs numériques.

\section{BIBLIOGRAPHIE}

[I) BICHSEL F.: Erfahrungstarifierung in der MotorfahrzeughaftpflichtVersicherung. Mitteilungen der Vereinigung Schweizer Versicherungsmathematiker 64 (I964), p. Ir9-130.

[2] Coppini M. A.: A propos de la distribution des cas de maladie entre les assurés et par rapport à la durée. The Astin Bulletin, Vol II, Part I (I962), P. 45-6I.

[3] Delaporte P. J.: Un problème de tarification de l'assurance accidents d'automobile examiné par la statistique mathématique. Comptes Rendus du XVIè Congrès International d'Actuaires, Vol II (I960), p. I 2 I-I35.

[4] D'Hooge L.: Studie van de heterogeniteit van een tariefklasse in niet levens-verzekeringen. De Wallens--Leuven (I968)

[5] D'Hooge L.: Décomposition d'une classe de tarif par post-optimation. Comptes Rendus du Igème Congrès International d'Actuaires à Oslo (I972), Abstract p. 228-229.

[6] DROPKIN L. B.: Size of Ioss distributions in workmen's compensation insurance. Proceedings of the Casualty Actuarial Society, Vol LI (1964); p. $198-259$.

[7] Franckx E.: Le problème de la classe de tarif et le choix d'une base de décomposition. Bulletin de Association Royale des Actuaires Belges 66 (I97I) ; p. 223-232.

[8] Gale D.: The theory of lineair economical models. Mc Graw-Hill (I960)

[9] Srmonnard: Programmation linéaire. Dunod-Paris (1962) 